\title{
Research on Technology of Electromagnetic Protection for the Generator Control System
}

\author{
Baocheng Wang, Guyong Han, Lin Zhu \\ Xuzhou Air Force College, Xuzhou, China \\ Email: xzkjxywangbaocheng@163.com
}

Received December 2013

\begin{abstract}
Generator control system decides whether the generator can work as usual or not, as well as its stability of performance. Both types of generators control system composed of the transistor and DSP are sensitive to outward electromagnetic interference, directly related to the generator performance. In this text, we first analyze the electromagnetic interference threat generator control system of transistor type may face, then design a electromagnetic protection plan for the intake, the panel and the sense organ. This work is of great significance in improving its electromagnetic protection ability and stability of performance.
\end{abstract}

\section{Keywords}

Generator Control System; Electromagnetic Interference Damage Analysis; Electromagnetic Protection Design

\section{Electromagnetic Interference Threat Analysis of Generator Control System}

Electromagnetic interference mainly gives an effect on the booster in control system. The principle and structure of transistor type are shown by Figure 1.

The drive unit composed of triangle wave producer, voltage comparer and driver amplifier circuit, output square wave $u_{b}$, as drive power switch. Triangle wave producer output triangle waves of certain value and frequency, without in-phase problem. Voltage comparer completes the square wave output $u_{a}$ after comparing trigonal wave with control voltage $u_{2}$ and amplifying the signal. The output is low voltage when the value of trigonal wave is less than $u_{2}$, while high voltage on the contrary, just as Figure 2 shows. Triangle wave producer and voltage comparer can be gained by operational amplifier circuit. After processed through power amplifying and opposite phase $u_{a}$ are translated as the drive signal $u_{b}$. The drive unit is also a proportional tache with the input $u_{2}$ and the output $u_{a}$.

With coupling interference signals to PID regulate circuit, the scope of trigonal wave changes or control voltage becomes uncertain which makes the value of ton/T (expressed as) out of gear, as is shown by Figure 3. Thus, the value of actual excitation current will be restricted by interference signals. What's more, uncertain value of interference signals result in excitation current regulate chaos, which cause excitation current out of control, then boosters abnormal voltage regulate, after that generators uncertain voltage output, finally power supply vehicle failure in airplanes start-up or offering electrifying check. 


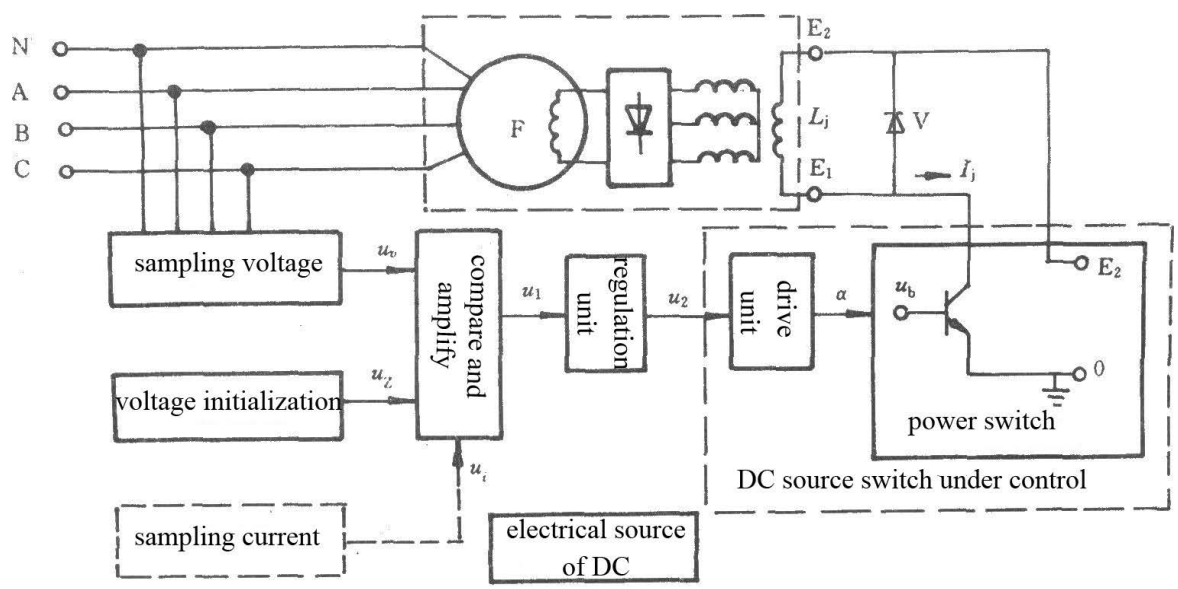

Figure 1. The system principle and structure frame diagram of transistor type.

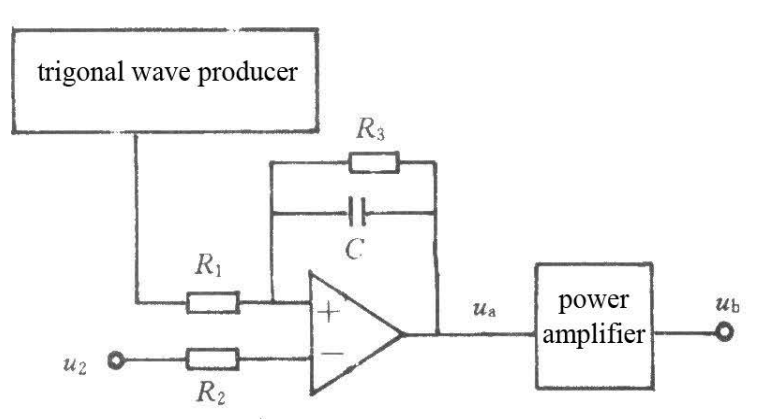

(a)

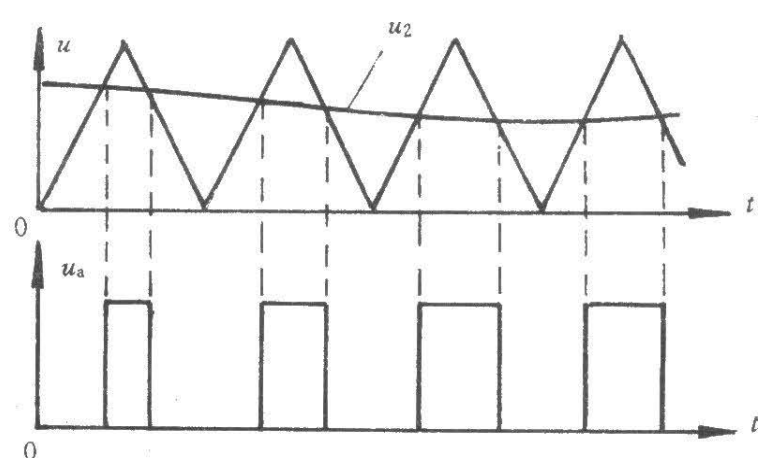

(b)

Figure 2. Structure and wave graph of the driving unit.
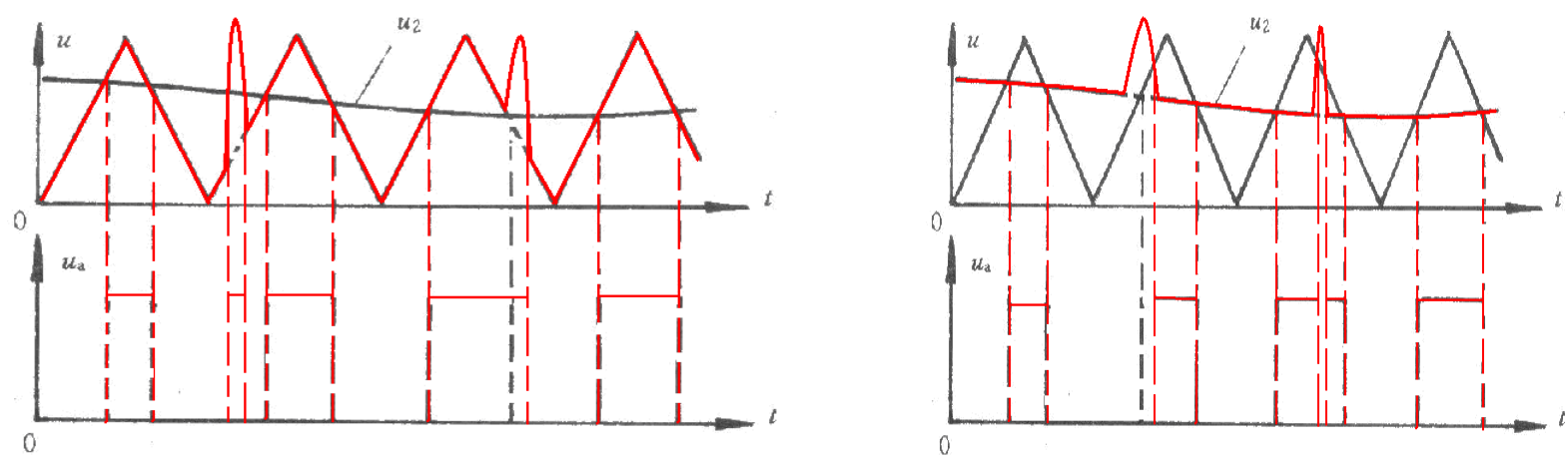

Figure 3. Compared with wave graphs interfered by signals.

\section{Research on Technology of Electromagnetic Protection for Generator Controls System}

According to the analysis and test results of electromagnetic interfere with generator of transistor type, we carry out a research on electromagnetic protection technique and design a plan for the generator control system.

\subsection{Shield Shell for the Intake}

Electric material is generally used in electromagnetic shield when the electromagnetic field is of high-frequency radiation, with both electric field and magnetic field existing at the same time. In high-frequency segment, well-conducted electric material can shield the inside components from electric field and magnetic field. 
Table 1 shows the characteristic of usual shield material. According to the relative electromagnetic protection grade, we choose ferromagnetic material of intact structure. For the thickness of the material and the way to dispose joint and grounding directly relative to the shield efficiency, what we must solve actually is the factors engendering electric discontinuity of shield shell, such as the intake, the display window, handling parts, cables drilling through shield shell and so on [1].

The cooling intake on generator, electric discontinuous, brings bad electromagnetic leakage. If we ignore the thickness of shell, its shield efficiency under the worst far electromagnetic field is as follows:

$$
S E=100-20 \lg D-20 \lg D \times f
$$

In this formula, $D$ expresses the bore diameter and $f$ expresses the frequency of incident electromagnetic wave. According to the formula, the electromagnetic leakage is involved with the size of the bore. The bigger size, the worse shield efficiency. So in the airway design, we replace the original bore by several smaller ones with same hatch area. We choose boring metal plank at low cost, as Figure 4 shows. But when these same bores arrange together regularly (an interval less than $\lambda / 2$ ), it will worsen the shield efficiency (to 20lg $N / 2$ ). Therefore, the bores design must be designed irregularly arranging with unequal mutual intervals, as is shown by Figure 5 .

\subsection{Grounding Protection for the Control Panel}

What the generator may suffer is high-frequency electromagnetic wave. Each control loop can be grounded as

Table 1. The characteristic of usual shield material.

\begin{tabular}{|c|c|c|c|c|}
\hline mental & silver & copper & aluminium & zinc \\
\hline relative conductivity $\sigma_{r}$ & 1.05 & 1.00 & 0.61 & 0.29 \\
\hline relative permeability $\mu_{r}$ & 1 & 1 & 1 & 1 \\
\hline mental & brass & nickel & iron & Chemin nickel-plate \\
\hline relative conductivity $\sigma_{r}$ & 0.26 & 0.20 & 0.17 & 0.02 \\
\hline relative permeability $\mu_{r}$ & 0.26 & 0.20 & 0.17 & 0.02 \\
\hline
\end{tabular}

Figure 4. Mental plank with original bores.

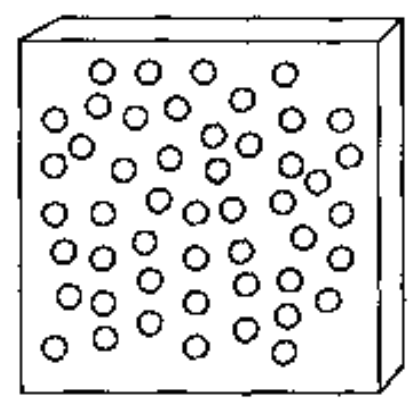

Figure 5. The design for the intake. 
Figure 6 shows. When some interferential coupling occurs, the interfere will be led to the earth for lower impedance of capacity at high frequency, preventing electronic components inside the generator from interference and impact.

\subsection{Install a filter in the Sensitive Part}

1) As Figure 7 shows. With the both coil ends $\left(E_{1} \sim E_{2}\right)$ shunt-wound the filter circuit included capacitor $C$ can absorb the upper parts of surge peak inside the excitation current, consequently weakening or eliminating the interference [2].

The reactance of capacitor $C$ is involved with frequency. Its logarithmic characteristic of breadth and frequency is as follows:

$$
20 \lg \mathrm{A}(\omega)=20 \lg \frac{1}{\omega C}=-20 \lg \omega C
$$

Obviously, with $\omega$ ranges from $2 \pi f$ to infinity, the effect of voltage attenuation is gradually enhanced. If the source resistance is as equal as load resistance, its insert ullage can be expressed as:

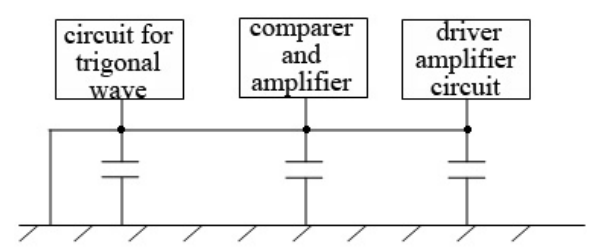

Figure 6. The sketch map of grounding.
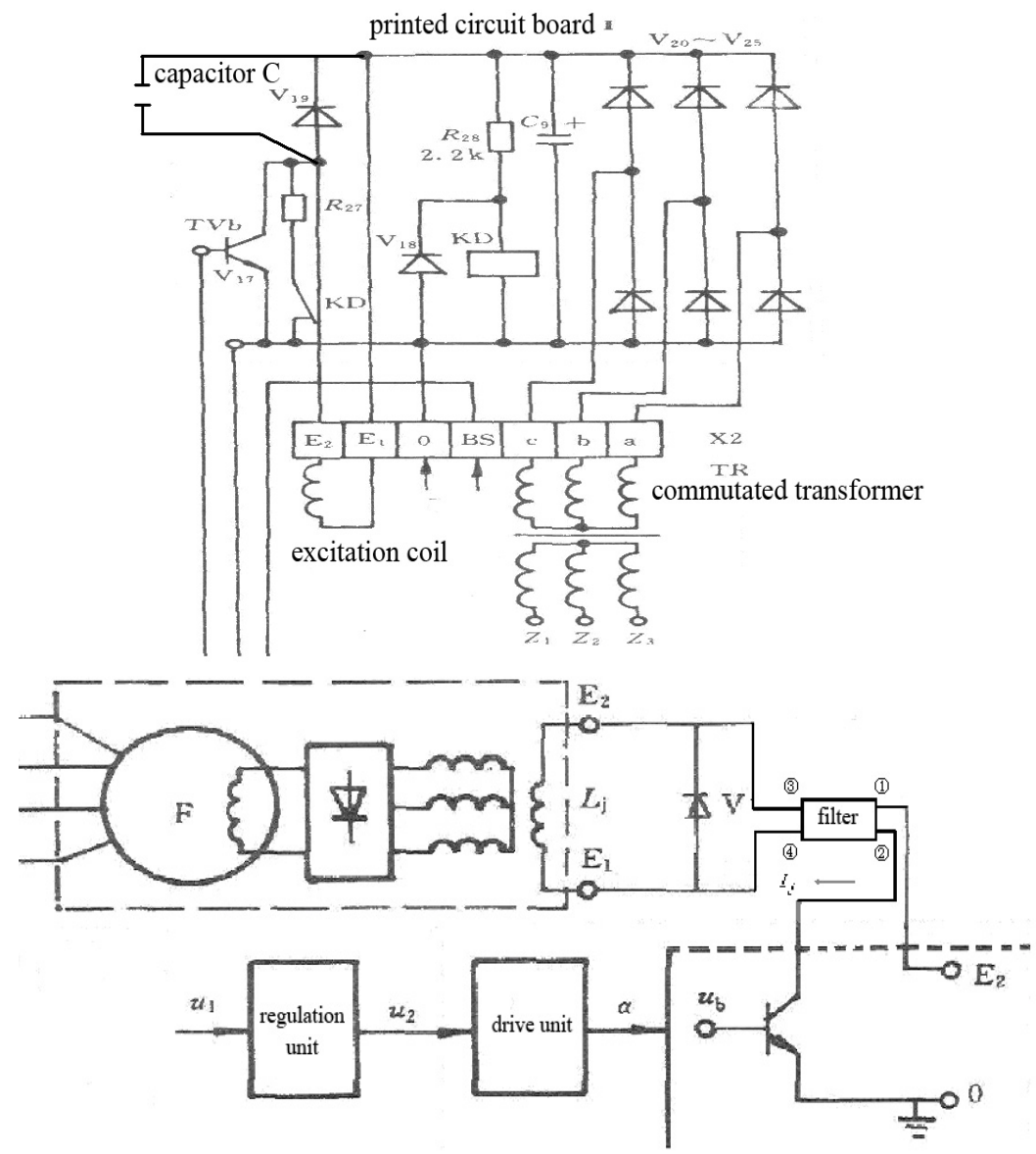

Figure 7. Safeguard for excitation winding. 


$$
L_{A}=10 \lg \left[1+(\pi f R C)^{2}\right]
$$

In this formula, $f$ expresses the work frequency $(\mathrm{Hz}), R$ expresses the resistance of source or load $(\Omega)$ and $C$ expresses capacitance $(\mathrm{F})$.

In order to make the power supply vehicle stable of performance under the complicated electromagnetic environment, the value of voltage attenuation is required at least $30 \mathrm{~dB}$ when the frequency reaches $1 \mathrm{kHz}$. If the resistance initialization of the excitation coil is $500 \Omega$, according to the formula above the valve of capacitance $C$ can be calculated as $20 \mu \mathrm{F}$.

2) Install an EMI filter ahead the excitation coil [3]

The disadvantage of filter circuit with single capacitor is no more than $6 \mathrm{~dB}$ attenuation velocity per each frequency span. While we fit single series-wound inductance and single shunt-wound capacitor together, a new type of filter called type $\mathrm{L}$, the value can reach as much as $12 \mathrm{~dB}$ per each frequency span. Because the excitation current is DC, we choose lowpass. The way of connection is shown by Figure 8.

Figure 9 shows the equivalence principle of erase differential-mode and common-mode interferential signal. Its logarithmic characteristic of breadth and frequency is as follows:

$$
20 \lg \mathrm{A}(\omega)=-20 \lg \sqrt{(\omega R C)^{2}+\left(1-\omega^{2} L C\right)^{2}}
$$

If $\omega_{0}=\frac{1}{\sqrt{L C}}$ and $\omega^{2} L C=\left(\frac{\omega}{\omega_{0}}\right)^{2}$, we can get the results as follows.

$$
20 \lg \mathrm{A}(\omega)=-40 \lg \frac{\omega}{\omega_{0}}
$$

When $\omega<<\frac{1}{\sqrt{L C}}$,

$$
20 \lg \mathrm{A}(\omega)=-20 \lg \sqrt{(\omega R C)^{2}+1} \approx 0
$$

When $\omega>>\frac{1}{\sqrt{L C}}$,

$$
\begin{aligned}
20 \lg \mathrm{A}(\omega) & =-20 \lg \sqrt{(\omega R C)^{2}+\left(\omega^{2} L C\right)^{2}} \\
& \approx-20 \lg \sqrt{\left(\omega^{2} L C\right)^{2}} \\
& =-20 \lg \omega^{2} L C
\end{aligned}
$$

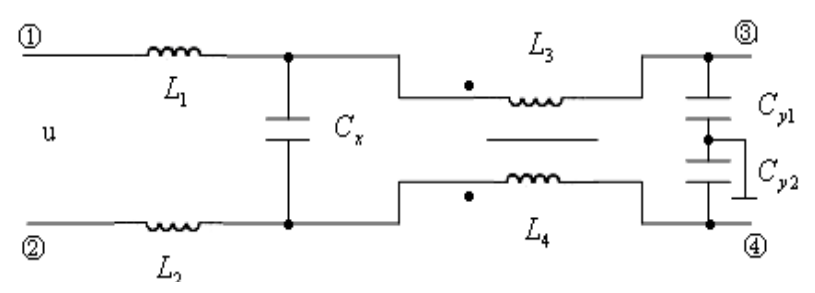

Figure 8. EMI filter.

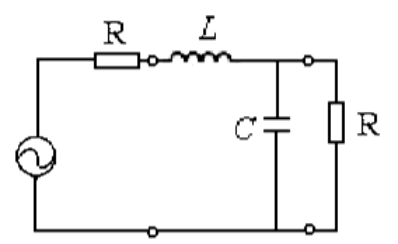

Figure 9. The equivalence principle of EMI filter. 
With $\omega$ ranges from $2 \pi f$ to infinity, the effect of voltage attenuation is gradually enhanced. If the source resistance is as equal as load resistance, its insert ullage can be expressed as:

$$
L_{A}=10 \lg \left\{\frac{1}{4}\left[\left(2-\omega^{2} L C\right)^{2}+\left(\omega R C+\frac{\omega L}{R}\right)^{2}\right]\right\}
$$

In this formula, $L$ expresses filter inductance $(\mathrm{H}), \omega=2 \pi f$, and terminate frequency $\quad f_{0}=\frac{1}{\pi \sqrt{2 L C}}$.

There are several ways to solve the initialization of $L$ and $C$ in order to reach the minimum value of voltage attenuation at the $1 \mathrm{kHz}$-frequency. For example, $L=1 \mathrm{mH}$, while $C=5 \mathrm{~F}$. In detail, $L_{1}=L_{2}=1 \mathrm{mH}, C_{x}=5 \mathrm{~F}$, $L_{3}=L=1 \mathrm{mH}_{4}$, and $C_{y 1}=C_{y 2}=5 F$.

\section{The Tag}

Research on technology of electromagnetic protection for generator controls system effectively solves the electromagnetic interference with the generator, keeping the performance stability of the generator under complicated electromagnetic environment. Particularly in the military realm, it is of great realistic significance in developing the ability of electromagnetic protection for generators and support capability of military equipment.

\section{References}

[1] Lv, W.H., Guo, Y.J., Tang, F.H., Yang, Y. and Chen, Y.F. (2008) Electromagnetic Compatible Principle and Application Tutorial (version 2). Tsinghua University Press, Beijing.

[2] Zhao, G. (2007) The Development Trend of Weapon’s Electromagnetic Compatible Technique. Electronic Engineering of the Warship, 20-22.

[3] Zhou, Z.M. and Ji, A.H. (2007) Electromagnetic Compatible Technique. Electronic Industry Press, Beijing. 